\title{
Manejo seguro de la nutrición enteral domiciliaria
}

\section{Safe management of home enteral nutrition}

\author{
Olga Lucia Pinzón-Espitiaº, Javier Leonardo González - Rodriguez² \\ Universidad Nacional de Colombia. Facultad de Medicina, Bogotá, Colombia. \\ Universidad del Rosario. Bogotá, Colombia. \\ * Dirigir correspondencia a:. olpinzone@unal.edu.co
}

\begin{tabular}{l} 
Proceso Editorial \\
\hline Recibido: 100820 \\
Aceptado: 190122 \\
Publicado: 160222 \\
\hline
\end{tabular}

DOI 10.17081/innosa.145

(C)Copyright 2022.

Pinzón O. et al.

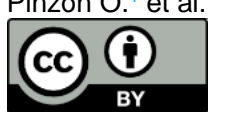

\begin{abstract}
RESUMEN
Introducción: Realizar un análisis de las prácticas en seguridad del paciente, en la atención nutricional en instituciones de salud domiciliaria (Homecare). Métodos: se realizó una revisión sistemática de la literatura. Para ello se hizo una búsqueda de artículos a través de las bases de datos PubMed®, ScienceDirect® y SciELO®, publicados en los últimos 10 años ( 1 de junio de 2010 - 1 de junio de 2020) en español, inglés y portugués que incluyeran temáticas relacionadas con la seguridad del paciente. Conclusiones y recomendaciones: La nutrición enteral en el hogar debe tener como objetivo mantener o mejorar el estado nutricional, y contribuir a una adecuada evolución clínica de los pacientes. Las recomendaciones identificadas corresponden a indicaciones relacionadas con procesos, manejo de tecnologías, calidad microbiológica y composición nutricional de las fórmulas nutricionales comerciales versus las dietas hechas en casa como criterios para garantizar la seguridad del paciente.

Palabras clave: Servicios de Atención de Salud a Domicilio; Nutrición Enteral; Seguridad del Paciente..
\end{abstract}

\begin{abstract}
Background: Perform an analysis of patient safety practices in nutritional care in hospital at home (Homecare). Methods: A systematic review of the literature was conducted. To do this, we searched for articles through the PubMed $\AA$, ScienceDirect $\AA$ and SciELO $\circledast$ databases, published in the last 10 years ( 1 June 2010 - 1 June 2020) in Spanish, English and Portuguese that included topics related to patient safety. Conclusions: Enteral nutrition at home should aim to maintain or improve nutritional status and contribute to an adequate clinical evolution of patients. The recommendations identified correspond to indications related to processes, technology management, microbiological quality and nutritional composition of commercial nutritional formulas versus home-made diets as criteria to guarantee patient safety.

Keywords: Enteral Nutrition; Home Care Services; Patient Safety
\end{abstract}

\section{INTRODUCCIÓN}

La seguridad del paciente como prioridad en los sistemas de salud a nivel mundial, se constituye en un reto que involucra múltiples actores, como son los aseguradores, los prestadores de servicios de salud, los pacientes y los cuidadores, quienes requieren de acciones particulares, como parte de los programas de mejoramiento continuo, para abordar de manera óptima y segura la atención médica (1). 
Complementando lo anterior, Padgett, Gossett (2) reportan que los errores médicos prevenibles resultan en la pérdida de 200,000 vidas por año con cargas financieras y operativas importantes para las organizaciones y la sociedad, siendo necesario trabajar en estrategias que permitan mejorar la seguridad y promover una cultura de atención segura al paciente para revertir dicha tendencia.

Hace aproximademente 20 años, el reporte denominado "To err is human: An Institute of Medicine report"'(3) mostró que entre 44,000 y 98,000 estadounidenses mueren cada año como resultado directo de errores médicos cometidos en hospitales, convirtiéndose en la octava causa principal de muerte, superando los accidentes de tránsito, el cáncer de seno y el SIDA, por lo que emitió recomendaciones enfocadas en establecer herramientas y protocolos para mejorar la base de conocimiento sobre seguridad, identificación y aprendizaje a partir de los errores, implementación acciones de supervisión, trabajo interdisciplinario y creación de sistemas de seguridad dentro de las organizaciones de atención médica para implementar prácticas seguras

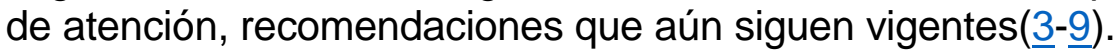

Dentro de las estrategias para una atención en el marco de la cultura de seguridad del paciente $(\underline{10}-13)$, el soporte nutricional enteral en el domicilio es referida como una terapia médica prioritaria, en la medida que propende por mantener o mejorar el estado nutricional fuera del ámbito hospitalario, por lo cual se debe contar con protocolos que incluyan recomendaciones de atención de acuerdo con los tiempos de recuperación, tipo de terapia nutricional, que favorezcan una mejor evolución, menor estancia hospitalaria, reducción de consumo de recursos y costos de atención (14-17).

Por lo tanto, las intervenciones terapéuticas deben incluir estrategias que garanticen la seguridad de los pacientes que reciben terapia de nutrición enteral, soportadas en una estructura de supervisión que avale la coherencia de las políticas, los procedimientos y las prácticas del proceso de apoyo nutricional, con el objetivo de lograr la reducción de riesgos soportados en las recomendaciones de mejores prácticas (18).

De este modo, en el marco de la atención nutricional en los cuidados domiciliarios, adicionalmente se deben establecer estrategias enfocadas en la "Cultura Justa", la cual es definida como un modelo de evaluación de la conducta de los proveedores con el fin de crear mejores resultados para los pacientes, cambiando el enfoque centrado en los errores y los resultados al diseño del sistema, a un enfoque preventivo y de incentivar buenas prácticas (19).

Asimismo, en la literatura se observa que dado el aumento en la prevalencia de la formulación de la nutrición enteral en el hogar, han surgido nuevas tendencias que incluyen diferentes tecnologías en salud, como los dispositivos para el paso de la alimentación enteral, las fórmulas nutricionales, las innovaciones prácticas y los desafíos con los reembolsos económicos $(\underline{20}, \underline{21})$.

\section{MÉTODOS}

2.1 Estrategia de búsqueda: Para la presente revisión sistemática de la literatura se realizó una búsqueda de artículos a través de las bases de datos PubMed®, ScienceDirect® y SciELO®, en los últimos 10 años ( 1 de junio de 2010 - 1 de junio de 2020). Las palabras clave utilizadas fueron en inglés (MeSH): "Enteral Nutrition", "Home Care Services", "Patient Safety". En español las palabras 
utilizadas para esta fueron (Decs): "Servicios de Atención de Salud a Domicilio", "Nutrición Enteral", "Seguridad del Paciente".

Se trata de una revisión sistemática de literatura que incluyó las siguientes etapas: elaboración de la pregunta de investigación, búsqueda de literatura, evaluación de información, análisis de datos a partir de criterios de división, exposición y comparación, y finalmente la presentación de los resultados (2224). En la presente revisión de literatura se siguieron las recomendaciones del protocolo PRISMA(2527).

2.2. Criterios de inclusión: Los criterios de inclusión de los estudios fueron los siguientes: responder a la pregunta de investigación, siendo principalmente estudios observacionales y publicados en los últimos diez años, con el fin de identificar practicas seguras sobre el tema en cuestión.

Para la selección de los artículos se estableció filtro por tipo de artículo, incluyendo ensayos clínicos, metaanálisis, ensayos controlados aleatorizados, revisiones y revisiones sistemáticas, publicados en los últimos 10 años, en idioma español, inglés y portugués.

2.3. Criterios de exclusión: Artículos de reflexión e informes de experiencias, así como las cartas al editor, revisiones integradoras de literatura, disertaciones, tesis, resúmenes de conferencias y editoriales de revistas sin características científicas fueron excluidas.

2.4. Selección de los estudios: Dos expertos en los temas de nutrición y seguridad del paciente realizaron de manera independiente la búsqueda y el análisis de los estudios, incluyendo en la matriz de análisis: título, resumen, introducción, métodos, resultados y discusión. Se realizó una cuidadosa selección de artículos según los criterios de elegibilidad, se sintetizó y ordenó según las variables relacionadas con la seguridad del paciente para la clasificación dese las recomendaciones en seguridad del paciente.

Posteriormente, las publicaciones seleccionadas en el anterior punto, fueron leídas en su totalidad. Finalmente, estas fueron releídas y analizadas de acuerdo con los criterios de elegibilidad, para así conformar la muestra final seleccionada.

Complementariamente, fue necesario comparar los resultados de los dos investigadores y establecer las diferencias, para ser resueltas por consenso, en el caso en que fue requerido un tercer revisor, este fue invitado al panel de discusión para favorecer la validación de la selección de estudios incluidos para el análisis.

La búsqueda en las bases de datos dio como resultado 59 publicaciones, que fueron seleccionados de acuerdo con los criterios de elegibilidad, lo que dio como resultado la muestra final de 11 artículos (Figura 1).

\section{RESULTADOS}

La búsqueda electrónica inicial permitió encontrar un total de 59 artículos con las palabras clave nutrición enteral y servicios de atención de salud a domicilio identificadas en las diferentes bases de datos ( 23 en PubMed®, 23 en ScienceDirect ${ }^{\circledR}$ y 13 en SciELO®). Una vez realizada la tamización por título, y descartar los estudios duplicados, se eliminaron 38 estudios, por no cumplir con los criterios de inclusión. A los 21 artículos restantes se les aplicó 
un segundo filtro de elegibilidad que consistió en la revisión del título y el resumen para corroborar si en el contenido de este se consideraban resultados relacionados con las palabras clave.

Figura 1. Diagrama de selección de los estudios incluidos en esta revisión integrativa

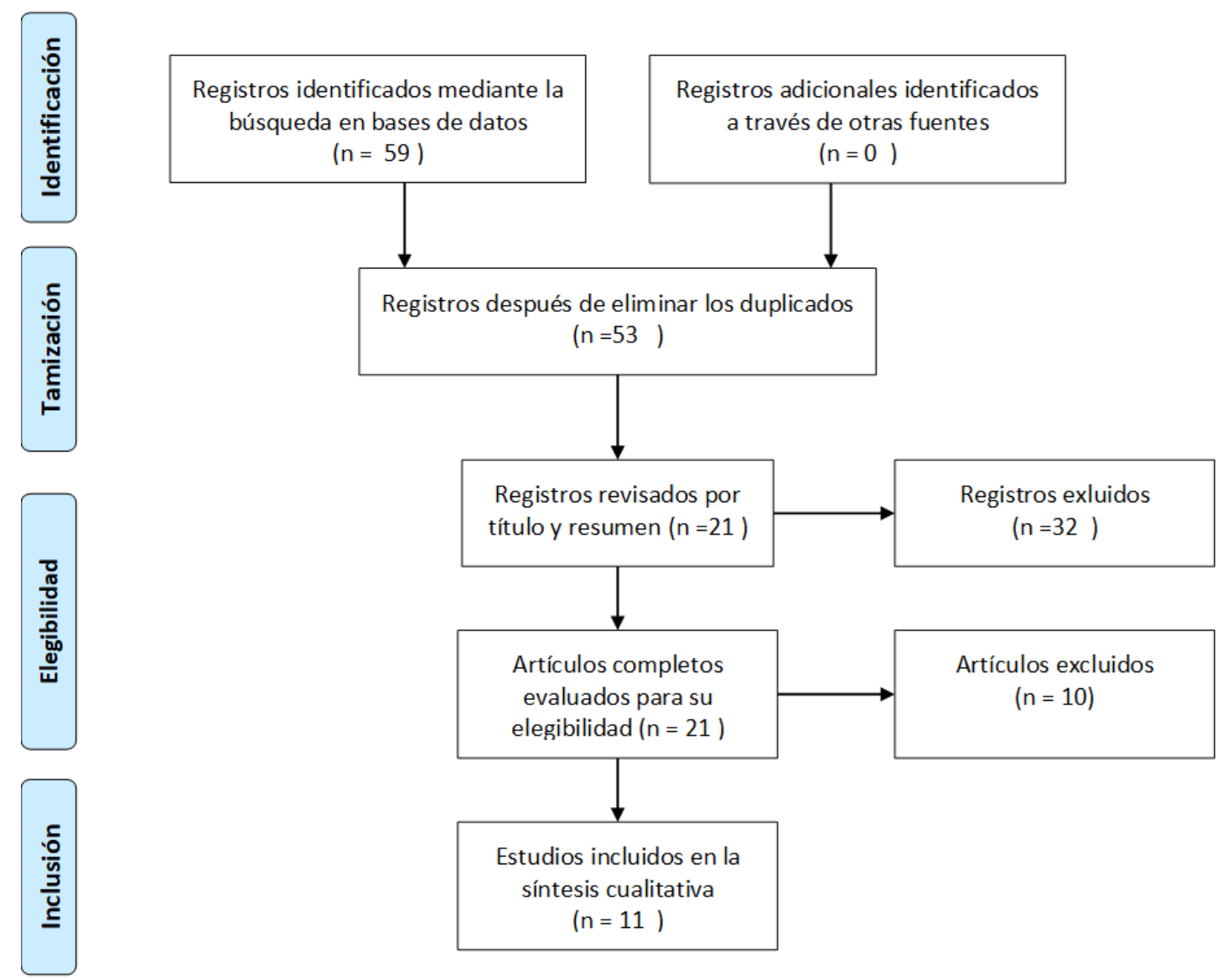

Fuente: Preferred Reporting Items for Systematic Reviews and Meta-Analyses: The PRISMA Statement $\underline{(56)}$.

Dado que los 21 cumplían criterios de elegibilidad, se les realizó lectura completa y minuciosa para identificar variables relacionadas con la seguridad del paciente, proceso que permitió la selección de 11 artículos y descartar 10 publicaciones (15, 16, 28-35). En la tabla 1 se muestra el resumen sistemático de los artículos seleccionados, que incluyen los siguientes datos: tema, año, titulo, tipo de estudio y objeto de estudio. 
Tabla 1. Resumen sistemático de los artículos seleccionados

\begin{tabular}{|c|c|c|c|c|}
\hline TEMA & AÑO & TITULO & $\begin{array}{c}\text { TIPO DE } \\
\text { ESTUDIO/DISEÑO }\end{array}$ & $\begin{array}{c}\text { POBLACION / } \\
\text { RECOMENDACIONES }\end{array}$ \\
\hline Procesos & 2020 & $\begin{array}{l}\text { ESPEN guideline on home enteral } \\
\text { nutrition }\end{array}$ & $\begin{array}{l}\text { Recomendaciones: } \\
\text { Opinión de expertos }\end{array}$ & 61 recomendaciones \\
\hline Procesos & 2019 & $\begin{array}{l}\text { ESPEN guideline on clinical nutrition } \\
\text { and hydration in geriatrics }\end{array}$ & $\begin{array}{l}\text { Recomendaciones: } \\
\text { Opinión de expertos }\end{array}$ & 81 recomendaciones \\
\hline Procesos & 2019 & $\begin{array}{l}\text { Estado nutricional e evolução clínica } \\
\text { de idosos em terapia nutricional } \\
\text { enteral domiciliar: uma coorte } \\
\text { retrospectiva }\end{array}$ & $\begin{array}{l}\text { Estudio observacional de } \\
\text { cohorte retrospectivo }\end{array}$ & 218 pacientes adultos \\
\hline Equipos & 2018 & $\begin{array}{l}\text { A Retrospective Comparative Study of } \\
\text { Continuous Pumping for Home } \\
\text { Enteral Nutrition } \\
\text { Esophagectomy }\end{array}$ & Estudio comparativo & $\begin{array}{l}108 \text { casos de pacientes } \\
\text { adultos con } \\
\text { esofagectomía }\end{array}$ \\
\hline Procesos & 2018 & $\begin{array}{l}\text { Pediatric Nasogastric Tubes in the } \\
\text { Home: Recommendations for Practice }\end{array}$ & Revisión de Literatura & NA \\
\hline $\begin{array}{l}\text { Calidad } \\
\text { nutricional y } \\
\text { microbiológica }\end{array}$ & 2018 & $\begin{array}{l}\text { Nutritional and microbiological quality } \\
\text { of commercial and homemade } \\
\text { blenderized whole food enteral diets } \\
\text { for home-based enteral nutritional } \\
\text { therapy in adults }\end{array}$ & Estudio transversal & $\begin{array}{l}66 \text { muestras de dietas } \\
\text { enterales comerciales y } \\
\text { no comerciales }\end{array}$ \\
\hline Equipos & 2017 & $\begin{array}{l}\text { Current Practices in Home } \\
\text { Management of Nasogastric Tube } \\
\text { Placement in Pediatric Patients: A } \\
\text { Survey of Parents and Homecare } \\
\text { Providers }\end{array}$ & Estudio descriptivo & $\begin{array}{l}144 \text { padres y } 66 \\
\text { proveedores domiciliaria } \\
\text { atención dom } \\
\text { (pacientes pediátricos) }\end{array}$ \\
\hline Procesos & 2017 & $\begin{array}{l}\text { Implementación de una vía clínica de } \\
\text { prescripción de nutrición enteral } \\
\text { domiciliaria de Murcia. Perfiles y } \\
\text { características muestrales }\end{array}$ & $\begin{array}{l}\text { Vía clínica } \\
\text { Diseño observacional y } \\
\text { ambispectivo }\end{array}$ & 345 pacientes \\
\hline $\begin{array}{l}\text { Calidad } \\
\text { nutricional y } \\
\text { microbiológica }\end{array}$ & 2017 & $\begin{array}{l}\text { Development of enteral homemade } \\
\text { diets for elderly persons receiving } \\
\text { home care and analysis of macro and } \\
\text { micronutrient composition }\end{array}$ & $\begin{array}{l}\text { Prueba de viscosidad, } \\
\text { estabilidad, olor y color, } \\
\text { determinación analítica } \\
\text { de la composición } \\
\text { química de los nutrientes } \\
\text { de las dietas. }\end{array}$ & $\begin{array}{l}3 \text { dietas caseras para } \\
\text { nutrición enteral }\end{array}$ \\
\hline Procesos & 2013 & $\begin{array}{l}\text { New electronic protocol for home } \\
\text { enteral nutrition therapy }\end{array}$ & $\begin{array}{l}\text { Construcción del } \\
\text { protocolo electrónico }\end{array}$ & $\begin{array}{l}1793 \text { registros de } \\
\text { pacientes }\end{array}$ \\
\hline Equipos & 2012 & $\begin{array}{l}\text { Home Enteral Tube Feeding in } \\
\text { Children With Inherited Metabolic } \\
\text { Disorders: A Review of Long-Term } \\
\text { Carer Knowledge and Technique }\end{array}$ & Estudio descriptivo & $\begin{array}{l}32 \text { cuidadores de niños } \\
\text { con trastornos } \\
\text { metabólicos hereditarios }\end{array}$ \\
\hline
\end{tabular}

Fuente: Elaboración propia.

Se logró identificar que aproximadamente, el $55 \%$ de los estudios realizados en nutrición enteral domiciliaria y seguridad del paciente corresponden a desarrollo de recomendaciones relacionadas con procesos, el $27 \%$ con manejo seguro de equipos y el $18 \%$ con análisis nutricional y calidad microbiológica de las fórmulas nutricionales y las dietas hechas en casa.

De los estudios relacionados con procesos, se evidencia la organización de protocolos electrónicos para el registro de la terapia de nutrición enteral en el domicilio (36), 
implementación de una vía clínica de prescripción de nutrición enteral (37), estado nutricional y evolución clínica (38), recomendaciones el manejo seguro de los equipos y sondas (39-42), buenas prácticas $(21,43)$, seguido del análisis de las fórmulas comerciales en relación a la calidad nutricional y la garantía de la calidad microbiológica(44, 45$)$.

\section{DISCUSIÓN}

En el marco de la seguridad del paciente se identifica la necesidad de establecer como puerta de entrada el desarrollo de un flujograma de cuidado nutricional (Figura 2), que incluya la implementación de registros electrónicos de la atención nutricional dentro de la historia clínica del proveedor de cuidados y nutrición domiciliaria con el fin de organizar, estructurar y administrar información relacionada con el tratamiento nutricional y su respectivo seguimiento.

Figura 2. Cuidado nutricional seguro en Home Care

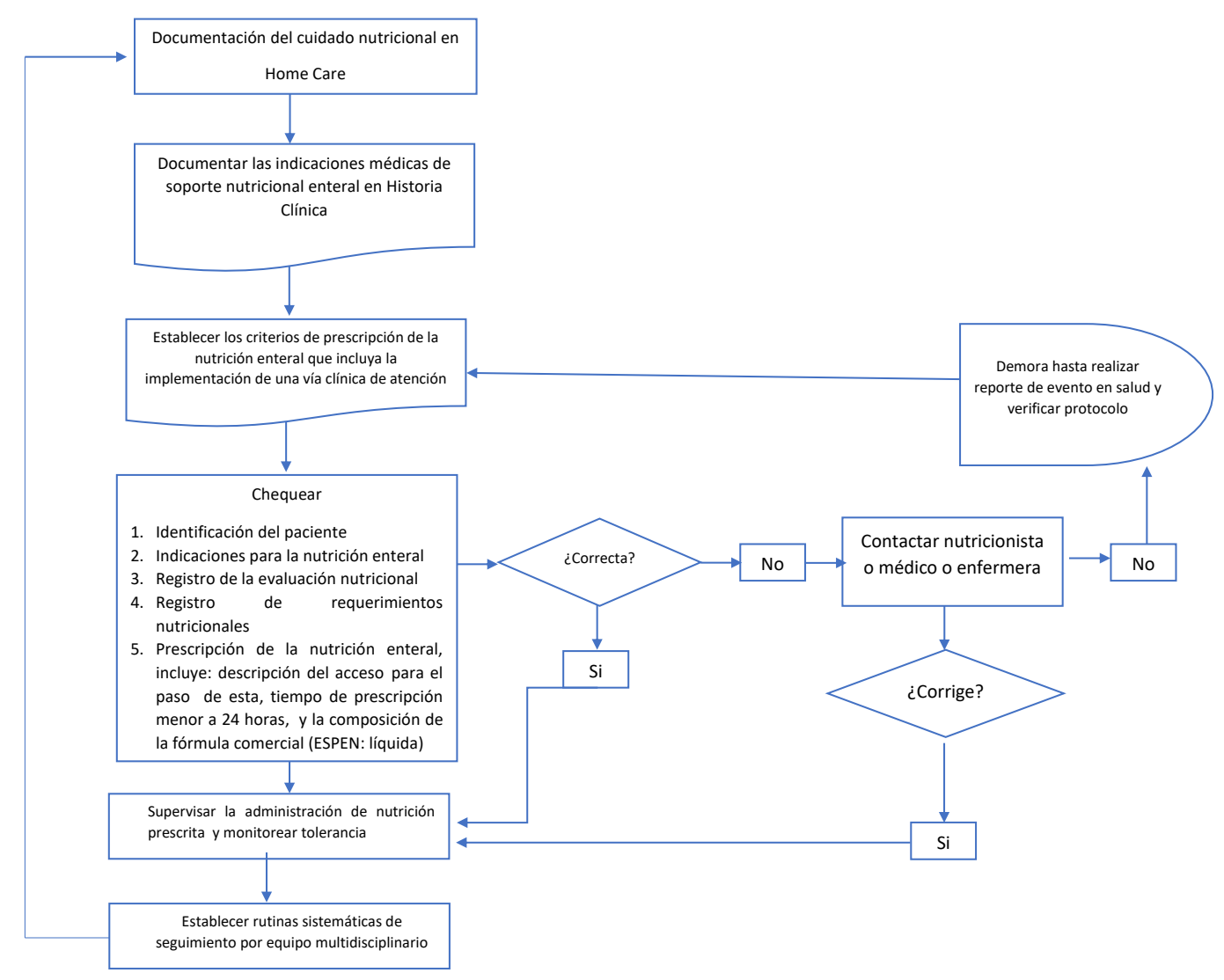

Fuente: Elaboración propia

Schieferdecker, Kuretzki (36) posterior a una revisión teórica sobre la terapia de nutrición enteral en el hogar en la que se identificaron variables de evaluación en el marco de la seguridad del paciente en la presente revisión, se observa que a partir de 1793 registros lograron establecer nueve categorías para identificar en una historia clínica nutricional buenas prácticas de registro, como por ejemplo, la identificación del paciente, la evaluación nutricional, la prescripción dietética, las indicaciones para la nutrición enteral, descripción del acceso para el paso de la nutrición enteral, la composición de la nutrición enteral, el sistema de 
administración utilizado, las complicaciones derivadas de la terapia y la identificación de readmisión.

Lo anterior se realizó mediante auditoria al Sistema Electrónico Integrado de Protocolos Computarizados (SINPE (C), en donde se identificaron los siguientes beneficios, impacto de la estandarización en la terapia nutricional en el desempeño de los miembros del equipo, mejoras en la calidad de la atención en la medida que permite garantizar buenas prácticas, permitiendo que el paciente sea tratado con mayor especificidad. Adicionalmente, reportan beneficios administrativos soportados en la literatura, como reducción del espacio físico requerido para archivar documentos, la cantidad de personas necesarias para la presentación, disminución de costos a largo plazo, facilitar la toma de decisiones a partir de captura de datos clínicos fiables $\underline{(36}, \underline{46}-\underline{-51})$.

Otra opción para tener en cuenta es la propuesta del desarrollo e implementación de una vía clínica de prescripción de nutrición enteral domiciliaria, que permita establecer el tratamiento nutricional de acuerdo con la patología de base, la vía de administración y el tipo de fórmula a utilizar, además de establecer estrategias que permitan controlar la adecuada prescripción del soporte nutricional (37).

Con relación a los dispositivos, la presente revisión, invita a garantizar buenas prácticas en torno al manejo de las sondas para nutrición enteral y las bombas para el paso de esta, teniendo en cuenta que el manejo de las sondas para paso de alimentación enteral en atención domiciliaria, es esencial para garantizar el suministro del volumen prescrito que permita cubrir los requerimientos nutricionales de los pacientes, por lo que se requieren de prácticas como, la verificación de la colocación de la sonda, la comprensión de los cuidados de las sondas por parte de los pacientes, las familias, los cuidadores y los proveedores de atención domiciliaria que brindan la atención directa con el paciente.

Las complicaciones mecánicas relacionadas con los dispositivos se constituyen en un problema en el entorno hospitalario, dificultades que se amplifican cuando los pacientes son remitidos o se encuentran en el entorno domiciliario, siendo esencial que el programa de hospitalización en casa cuente con equipos multidisciplinarios enfocados en el seguimiento de los pacientes para optimizar los resultados $\underline{(52)}$.

Es importante resaltar entonces, a partir de la publicación de Northington, Lyman (39) derivada de una investigación en población pediátrica, que incluyó 144 padres y 66 proveedores de atención domiciliaria, la cual a partir de los hallazgos relacionados con prácticas inadecuadas respecto a la verificación de la posición de la sonda y los cuidados correspondientes, confirman la necesidad de una práctica consistente entre los profesionales de la salud y en la educación de los padres, para garantizar la seguridad del paciente. Estos autores, por ejemplo, identificaron una práctica que ha sido reevaluada como lo es la auscultación para la confirmación de la ubicación de la sonda nasogástrica y, que aún se sigue usando ampliamente en la práctica clínica, a pesar de las alertas que no recomiendan su uso.

A la recomendación anterior los autores, en otra publicación teniendo en cuenta que la adecuada colocación de la sonda es uno de los dilemas que enfrentan las enfermeras de atención médica domiciliaria, plantean la discusión acerca de cuáles son las mejores prácticas 
para la colocación, verificación y prevención de complicaciones fuera del entorno de atención aguda, entornos en los que no se dispone de la radiografía (estándar de oro) para confirmar la colocación de acuerdo con la Alerta de Seguridad del Paciente y por la Sociedad Estadounidense de Nutrición $\underline{(42}, \underline{53)}$.

Por consiguiente, Northington L y colaboradores (39), destacan el papel de la enfermera como miembro del equipo de cuidado nutricional multidisciplinario para garantizar la seguridad del paciente en el hogar, a través la educación a pacientes, familias y cuidadores, así como la implementación de intervenciones basadas en evidencia para el hogar que les permitan identificar e implementar estándares y pautas de mejores prácticas.

Recomendación similar realiza Evans, Preston (41), sugiriendo reevaluar anualmente los conocimientos en alimentación por sonda enteral en el hogar de los cuidadores, en con un reentrenamiento obligatorio si no se demuestran las competencias básicas, dado que es frecuente identificar malas prácticas que pueden causar contaminación del alimento, concentración incorrecta, intolerancia, riesgos de aspiración, peritonitis e incluso descompensación metabólica.

Otro aspecto relevante, es el uso de bombas de infusión en los entornos domiciliarios y hospitalarios para garantizar practicas seguras mediante la implementación de estos equipos como barreras de seguridad. Dai, Fu (40) con el objetivo de discutir el efecto y la seguridad del paso de nutrición enteral por bomba por el método de infusión continua en el hogar después de la esofagectomía, analizaron 108 casos, de pacientes con una edad promedio de 62 años, de los cuales 56 pacientes recibieron alimentación con bomba y 52 pacientes alimentación por sonda tradicional, reportaron que al verificar la seguridad del paciente en el grupo que recibió alimentación con bomba de infusión fue significativamente mejor evidenciada a través de indicadores descritos a continuación.

Estos indicadores son, menor número de complicaciones dentro de los 2 meses posteriores al alta, en relación al indicador del mantenimiento del peso, la pérdida de peso dentro de las 4 semanas posteriores al alta fue de $0.13 \mathrm{~kg}$ frente a $3.90 \mathrm{~kg}$ en los pacientes que no contaban con bomba, esto asociado a tasas de cumplimiento de metas nutricionales significativamente más altas, que permiten evidenciar como estos equipos son un factor contribuyente para mejorar la tolerancia a la alimentación y su vez contribuyen a garantizar la efectividad del soporte nutricional (40).

En el marco de un tratamiento nutricional desde los reportes de literatura se sugiere contar con un protocolo de prescripción de la nutrición enteral, que incluya la verificación de la calidad microbiológica y el análisis de la composición nutricional de las fórmulas nutricionales vs patología para las cuales son recetadas, como indicador de seguridad, y así poder identificar los riesgos relacionados con el cumplimiento de metas nutricionales y de contaminación, los cuales son de alta prioridad en la atención y cuidado de los pacientes.

Un protocolo de seguridad en la prescripción de la nutrición enteral en los entornos de cuidados domiciliarios debe propender por prevenir estados de malnutrición o desnutrición (54), a través de estrategias de intervención que garanticen el mantenimiento y / o mejoría del estado clínico 
y nutricional, de manera que se pueda contribuir a una mejor evolución clínica y nutricional (38).

Con el objetivo de evaluar la calidad nutricional y microbiológica de las fórmulas nutricionales comerciales y los licuados de alimentos hechos en casa para ser administrados a pacientes adultos requirentes de terapia nutricional domiciliaria, Vieira, Santos $(44)$ realizaron un estudio transversal, que incluyó 33 muestras de fórmulas enterales comerciales y 33 licuados no comerciales en los hogares de pacientes que recibían terapia nutricional enteral en el hogar. De las 33 muestras comerciales, el 66,7\% fueron en presentación líquida $(n=20)$ y el $33.3 \%$ en presentación polvo $(n=13)$.

Tabla 2. Calidad nutricional y microbiológica de las fórmulas comerciales para nutrición enteral en casa

\begin{tabular}{|c|c|c|c|}
\hline \multicolumn{4}{|c|}{ Calidad nutricional } \\
\hline \multirow[t]{2}{*}{ Macronutrientes } & Proteína & Grasas & Carbohidratos \\
\hline & $(g / 100 g)$ & $(g / 100 g)$ & $(g / 100 g)$ \\
\hline Formula comercial - Líquida & 4.1 & 3.0 & 11.6 \\
\hline Formula comercial - Polvo & 4.1 & 4.3 & 13.2 \\
\hline Licuados caseros & 2.1 & 1.9 & 3.4 \\
\hline \multicolumn{4}{|c|}{ Calidad nutricional } \\
\hline \multirow[t]{2}{*}{ Micronutrientes y otros } & Minerales & Fibra dietética & Agua \\
\hline & $(g / 100 g)$ & $(g / 100 g)$ & $(g / 100 g)$ \\
\hline Formula comercial - Líquida & 0.6 & 1.9 & 79.2 \\
\hline Formula comercial - Polvo & 0.7 & 1.2 & 75.7 \\
\hline Licuados caseros & 0.7 & 0.8 & 91.1 \\
\hline \multicolumn{4}{|c|}{ Calidad microbiológica } \\
\hline $\begin{array}{l}\text { Formación de colonias } \\
\text { unidad } / \mathrm{mL}\end{array}$ & Mesófilos & Coliformes & Escherichia coli \\
\hline \multirow[t]{2}{*}{ Formula comercial - Líquida } & 0 & 0 & 0 \\
\hline & $0-0$ & $0-0$ & $0-0$ \\
\hline \multirow[t]{2}{*}{ Formula comercial - Polvo } & 360 & 180 & 0 \\
\hline & $5-15,000$ & $0-1,400$ & $0-0$ \\
\hline \multirow[t]{2}{*}{ Licuados caseros } & 40,000 & 14,000 & 0 \\
\hline & $3,000-2,400,000$ & $330-100,000$ & $0-5$ \\
\hline
\end{tabular}

Fuente: Vieira, Santos_(44).

Las muestras fueron analizadas en laboratorio para evaluar su calidad nutricional y microbiológica, y como indicadores del estudio se realizó seguimiento adicionalmente a los 
datos antropométricos de la circunferencia media del brazo y el grosor del pliegue cutáneo del tríceps a partir de los registros médicos de los pacientes, evidenciando que las fórmulas no comerciales (licuados hechos en casa) presentaron una calidad nutricional deficiente (44).

Lo anterior se afirma a partir de la evidencia de valores significativamente más bajos para proteínas, grasas, fibra, carbohidratos y energía, constituyéndose en un factor de riesgo para los pacientes, ya que los valores medidos para macronutrientes y energía correspondieron a menos del $50 \%$ de los valores prescritos (a excepción de la grasa). En contraste con las fórmulas comerciales en las cuales el valor de la proteína fue aproximadamente un $20 \%$ más que el valor prescrito, carbohidratos del $92 \%$, y los valores de grasa y energía correspondieron a aproximadamente el $100 \%$ de la prescripción como se puede ver en la tabla 2 (44).

En relación con la calidad microbiológica, y previa toma de muestras en el hogar de los pacientes antes de la administración de los licuados hechos en casa para alimentación enteral, los análisis reportaron altos niveles de contaminación bacteriana, ya que solo el $6.0 \%$ cumplió con el estándar para bacterias coliformes, detectándose Escherichia coli en 10 muestras de los licuados y en 2 formulas comerciales en polvo (44).

El estudio inmediatamente anterior, reportó que independientemente del tipo de dieta, la prevalencia de desnutrición fue alta en ambos grupos, siendo mayor en los pacientes que recibieron licuados.

Por otra parte, Jansen, Generoso (45) con el objetivo de realizar el análisis de la composición de macro y micronutrientes de las dietas enterales caseras, desarrollaron una dieta enteral casera estándar con tres diferentes densidades calóricas (1500, 1800 y $2100 \mathrm{Kcal}$ ), para posterior realización de pruebas de viscosidad, estabilidad, olor y color y composición química analítica de los nutrientes, reportando deficiencias de minerales y vitaminas como por ejemplo el magnesio, las vitaminas $\mathrm{E}, \mathrm{D}$ y $\mathrm{B} 6$, requiriendo ser complementadas para las opciones de 1800 calorías, mientras que la de $1500 \mathrm{Kcal}$ no fue nutricionalmente segura en términos de micronutrientes.

También se observó, en un estudio observacional que incluyó 203 pacientes con una edad promedio 52.5 años que recibieron dietas caseras durante al menos 12 meses antes de comenzar un programa especializado de nutrición en el hogar, y en los 12 meses siguientes se les giró a fórmulas enterales comerciales acompañados por un equipo de soporte nutricional, que esta última intervención redujo significativamente el número de ingresos hospitalarios, disminución de las prevalencia de complicaciones y del costo promedio anual del tratamiento hospitalario el cual pasó de \$ 764.65 por paciente a \$142.66 por año por paciente(55).

Teniendo en cuenta lo anterior, en el entorno domiciliario se debe evaluar la adecuada prescripción de fórmulas nutricionales que permita cubrir las necesidades nutricionales de los pacientes, de acuerdo con la recomendación de la Sociedad Europea de Nutrición Enteral y Parenteral - ESPEN - sobre nutrición enteral en el hogar, recomienda utilizar alimentación por sonda enteral con fórmula comercial estándar, ya que las mezclas hechas en casa (licuados) aunque son menos costosas, son menos efectivas y seguras que las fórmulas para nutrición 
enteral producidas comercialmente, por lo que precisan que estas no deben utilizarse en pacientes con indicación de nutrición enteral domiciliaria(21, $\underline{56)}$.

Adicionalmente ESPEN recomienda monitorear la eficacia a partir de indicadores como el peso corporal, la composición corporal y el estado de hidratación, e incluir mediciones de laboratorio, como albúmina sérica o transferrina, y la monitorización de las complicaciones asociadas al cuidado de la sonda y la administración (21).

En el 2019, la guía de ESPEN sobre nutrición e hidratación clínica en geriatría, recomienda garantizar un aporte de ingesta de proteínas de al menos $1 \mathrm{~g}$ por kilogramo de peso corporal al día, ajustadas a las necesidades individuales teniendo en cuenta el estado nutricional, el nivel de actividad física, el estado de la enfermedad y la tolerancia, así como de los micronutrientes de acuerdo con la recomendación para las personas mayores sanas (43), por lo cual se debe tener cuidado en la elección de la fórmula nutricional a seleccionar para prevenir riesgos en este grupo poblacional.

\section{CONCLUSIONES}

La nutrición enteral en instituciones de salud domiciliaria en el marco de la cultura de la seguridad del paciente, debe propender por la implementación de procesos de cuidado nutricional seguros en entornos domiciliarios, que incluyan la implementación y verificación de protocolos de atención nutricional, dirigidos por equipos interdisciplinarios con formación en soporte nutricional enteral, que focalicen su atención no solo en el manejo seguro de los dispositivos, sino también en garantizar la calidad nutricional y microbiológica de las fórmulas nutricionales, para favorecer adecuados desenlaces clínicos y una evolución nutricional satisfactoria. Si bien en los artículos analizados se utilizan diferentes metodologías, los resultados que presentan concuerdan en que la nutrición enteral, debe propender por mantener o mejorar el estado nutricional fuera del ámbito hospitalario, esta debe estar basada en la implementación de altos estándares de calidad y pautas para las mejores prácticas de atención.

Contribución de los autores: Conceptualización, O. L. P. E.; investigación, O. L. P. E. y J. L R. G.; recursos, No aplica; escritura: preparación del borrador original, O. L. P. E.; escritura: revisión y edición, O. L. P. E.; visualización, O. L. P. E.; supervisión, J. L R. G.; adquisición de fondos, No aplica. Todos los autores han leído y aceptado la versión publicada del manuscrito.

Fondos: Esta investigación no recibió fondos externos.

Conflictos de intereses: Los autores declaran no tener ningún conflicto de intereses.

\section{REFERENCIAS}

1. Dixon-Woods M, Pronovost PJ. Patient safety and the problem of many hands. BMJ Qual Saf. 2016;25(7):485-8. DOI:10.1136/bmjgs-2016-005232

2. Padgett J, Gossett K, Mayer R, Chien W-W, Turner F. Improving Patient Safety through High Reliability Organizations. Qualitative Report. 2017;22(2). DOI:10.46743/2160-3715/2017.2547 
3. Wakefield M. To err is human: An Institute of Medicine report. Professional Psychology: Research and Practice. 2000;31(3):243-244. DOI:10.1037/h0092814

4. Leape LL, Berwick DM. Five years after To Err Is Human: what have we learned? Jama. 2005;293(19):2384-90. DOI:10.1001/jama.293.19.2384

5. Havens DH, Boroughs L. "To err is human": a report from the Institute of Medicine. Journal of pediatric health care. 2000;14(2):77-80. https://pubmed.ncbi.nlm.nih.gov/10736144/

6. Bowdler NC. Quality of Care and Patient Safety. Proceedings in Obstetrics and Gynecology. 2019;9(2):1-2. Doi: 10.17077/2154-4751.1469

7. Gould J.C. (2019) The Institute of Medicine and the Pursuit of Quality. In: Morton J., Brethauer S., DeMaria E., Kahan S., Hutter M. (eds) Quality in Obesity Treatment. Springer, Cham. Doi:10.1007/978-3-030-25173-4_4

8. Mueller BU, Neuspiel DR, Fisher ERS. Principles of pediatric patient safety: Reducing harm due to medical care. Pediatrics. 2019;143(2):e20183649.Doi: 10.1542/peds.2018-3649

9. Afroza S. Patient Safety is the Priority for Quality Health Care. Journal of Bangladesh College of Physicians and Surgeons. 2020;38(1):1-2.

10. Weaver SJ, Lubomksi LH, Wilson RF, Pfoh ER, Martinez KA, Dy SM. Promoting a culture of safety as a patient safety strategy: a systematic review. Annals of internal medicine. 2013;158(5_Part_2):369-74. Doi: 10.7326/0003-4819-158-5-201303051-00002

11. Gartshore E, Waring J, Timmons S. Patient safety culture in care homes for older people: a scoping review. BMC health services research. 2017;17(1):752. Doi: 10.1186/s12913-017-27132

12. O'Donovan R, Ward M, De Brún A, McAuliffe E. Safety culture in health care teams: A narrative review of the literature. Journal of nursing management. 2019;27(5):871-83. Doi: 10.1111/jonm. 12740

13. Berland A, Holm AL, Gundersen D, Bentsen SB. Patient safety culture in home care: experiences of home-care nurses. Journal of Nursing Management. 2012;20(6):794-801. Doi: 10.1111/j.13652834.2012.01461.x

14. Wanden-Berghe C, Campos C, Burgos R, Álvarez J, Frías L, Matía P, et al. Registro del Grupo NADYA-SENPE de Nutrición Enteral Domiciliaria en España; años 2016 y 2017. Nutrición Hospitalaria. 2019;36(1):233-7. Doi:10.20960/nh.970

15. Wong A, Goh G, Banks M, Bauer J. A systematic review of the cost and economic outcomes of home enteral nutrition. Clinical Nutrition. 2018;37(2):429-42. Doi: 10.1016/j.clnu.2017.06.019

16. Klek S, Hermanowicz A, Dziwiszek G, Matysiak K, Szczepanek K, Szybinski P, et al. Home enteral nutrition reduces complications, length of stay, and health care costs: results from a multicenter study. The American journal of clinical nutrition. 2014;100(2):609-15. Doi: 10.3945/ajcn.113.082842

17. Dinenage S, Gower M, Van Wyk J, Blamey A, Ashbolt K, Sutcliffe M, et al. Development and evaluation of a home enteral nutrition team. Nutrients. 2015;7(3):1607-17. Doi: 10.3390/nu7031607 
18. Boullata JI. Safe practices for enteral and parenteral nutrition. In: Seres D., Van Way, III C. (eds) Nutrition Support for the Critically III: Springer; 2016. p. 229-41.Doi: 10.1007/978-3-319-21831$1 \_13$

19. Marx D. Patient Safety and the Just Culture. Obstetrics and gynecology clinics of North America. 2019;46(2):239-45.Doi:10.1016/j.ogc.2019.01.003

20. Martin K, Gardner G. Home enteral nutrition: updates, trends, and challenges. Nutrition in Clinical Practice. 2017;32(6):712-21.Doi: 10.1177/0884533617701401

21. Bischoff SC, Austin P, Boeykens K, Chourdakis M, Cuerda C, Jonkers-Schuitema C, et al. ESPEN guideline on home enteral nutrition. Clinical Nutrition. 2020;39(1):5-22. Doi:10.1016/j.clnu.2019.04.022

22. Whittemore R, Knafl K. The integrative review: updated methodology. Journal of advanced nursing. 2005;52(5):546-53. Doi: 10.1111/j.1365-2648.2005.03621.x

23. Hopia H, Latvala E, Liimatainen L. Reviewing the methodology of an integrative review. Scandinavian Journal of Caring Sciences. 2016;30(4):662-9.Doi: 10.1111/scs.12327

24. Souza MTd, Silva MDd, Carvalho Rd. Integrative review: what is it? How to do it? Einstein (São Paulo). 2010;8(1):102-6. Doi: 10.1590/s1679-45082010rw1134

25. Moher D, Shamseer L, Clarke M, Ghersi D, Liberati A, Petticrew M, et al. Preferred reporting items for systematic review and meta-analysis protocols (PRISMA-P) 2015 statement. Syst rev. 2015;4(1):1. Doi:10.1186/2046-4053-4-1

26. Shamseer L, Moher D, Clarke M, Ghersi D, Liberati A, Petticrew M, et al. Preferred reporting items for systematic review and meta-analysis protocols (PRISMA-P) 2015: elaboration and explanation. Bmj. 2015;349. Doi:10.1136/bmj.i4086

27. Moher D, Stewart L, Shekelle P. Implementing PRISMA-P: recommendations for prospective authors. Springer; 2016. Moher, D., Stewart, L. \& Shekelle, P. Implementing PRISMA-P: recommendations for prospective authors. Syst Rev, 2016;11. Doi:10.1186/s13643-016-0191-y

28. Bonnes SL, Salonen BR, Hurt RT, McMahon MT, Mundi MS. Parenteral and enteral nutritionfrom hospital to home: will it be covered? Nutrition in Clinical Practice. 2017;32(6):730-8. $10.1177 / 0884533617734491$

29. Vallumsetla N, Epp L, Hurt RT, Mundi MS. Effect of Home Enteral Nutrition on Diabetes and Its Management. Nutr Clin Pract. 2019;34(2):250-6. Doi: 10.1002/ncp.10104

30. Zeng J, Hu J, Chen Q, Feng J. Home enteral nutrition's effects on nutritional status and quality of life after esophagectomy. Asia Pacific journal of clinical nutrition. 2017;26(5):804. Doi:10.6133/apjcn.112016.07

31. Johnson TW, RN SS, Epp L, Mundi MS. Addressing frequent issues of home enteral nutrition patients. Nutr Clin Pract. 2019;34(2):186-95. Doi:10.1002/ncp.10257

32. Ireton-Jones C, Nishikawa K, Nishikawa R. Home parenteral and enteral nutrition during natural disasters: a guide for clinicians and consumers. Nutr Clin Pract. 2019;34(2):216-9. Doi:10.1002/ncp.10260

33. Ojo $\mathrm{O}$. The challenges of home enteral tube feeding: a global perspective. Nutrients. 2015;7(4):2524-38. Doi: 10.3390/nu7042524 
34. Ojo O. Pumps, feed and sets: is procurement limiting outcomes? Br J Nurs. 2015;24(3):146-9. Doi:10.12968/bjon.2015.24.3.146

35. Orlandoni P, Jukic Peladic N, Spazzafumo L, Venturini C, Cola C, Sparvoli D, et al. Utility of video consultation to improve the outcomes of home enteral nutrition in a population of frail older patients. Geriatr Gerontol Int. 2016;16(6):762-7. Doi:10.1111/ggi.12551

36. Schieferdecker M, Kuretzki C, Campos A, Malafaia O, Pinto J, Nascimento N. New electronic protocol for home enteral nutrition therapy. Arg Bras Cir Dig. 2013;26(3):195-9. Doi: 10.1590/s0102-67202013000300008

37. Ferrer-Gómez M, Sánchez-Romera J-F, García-Zafra M-V, Cuenca-Sánchez J-R, HernándezCascales A-B, Aranda-García A, et al. Implementación de una vía clínica de prescripción de nutrición enteral domiciliaria de Murcia. Perfiles y características muestrales. Nutr Hosp. 2017;34(3):517-23. Doi:10.20960/nh.839

38. Menezes CS, Fortes RC. Estado nutricional e evolução clínica de idosos em terapia nutricional enteral domiciliar: uma coorte retrospectiva. Rev Lat Am Enfermagem. 2019;27. Doi: 10.1590/1518-8345.2837.3198

39. Northington L, Lyman B, Guenter P, Irving SY, Duesing L. Current practices in home management of nasogastric tube placement in pediatric patients: a survey of parents and homecare providers. J pediatr nurs. 2017;33:46-53. Doi: 10.1016/j.pedn.2017.01.005

40. Dai L, Fu H, Kang X, Yang Y, Zhou H, Liang Z, et al. A retrospective comparative study of continuous pumping for home enteral nutrition after esophagectomy. Zhonghua wai ke za zhi [Chinese journal of surgery]. 2018;56(8):607-10. Doi:10.3760/cma.j.issn.0529-5815.2018.08.011

41. Evans S, Preston F, Daly A, Ashmore C, Holden C, MacDonald A. Home enteral tube feeding in children with inherited metabolic disorders: a review of long-term carer knowledge and technique. J Hum Nutr Diet. 2012;25(6):520-5. Doi: 10.1111/j.1365-277x.2012.01274.x

42. Northington L, Lyman B, Moore C, Guenter P. Pediatric Nasogastric Tubes in the Home: Recommendations for Practice. Home Healthc Now. 2018;36(3):148-53. Doi:10.1097/nhh.0000000000000650

43. Volkert D, Beck AM, Cederholm T, Cruz-Jentoft A, Goisser S, Hooper L, et al. ESPEN guideline on clinical nutrition and hydration in geriatrics. Clin Nutr. 2019;38(1):10-47. Doi:10.1097/nhh.0000000000000713

44. Vieira MMC, Santos VFN, Bottoni A, Morais TB. Nutritional and microbiological quality of commercial and homemade blenderized whole food enteral diets for home-based enteral nutritional therapy in adults. Clinical Nutrition. 2018;37(1):177-81. Doi: 10.1016/j.clnu.2016.11.020

45. Jansen AK, Generoso SdV, Guedes EG, Rodrigues AM, Miranda LAVdO, Henriques GS. Development of enteral homemade diets for elderly persons receiving home care and analysis of macro and micronutrient composition. Rev. bras. geriatr. gerontol.. 2017;20(3):387-97. Doi:10.1590/1981-22562017020.160168

46. Dorr D, Bonner LM, Cohen AN, Shoai RS, Perrin R, Chaney E, et al. Informatics systems to promote improved care for chronic illness: a literature review. J Am Med Inform Assoc. 2007;14(2):156-63. Doi: 10.1197\%2Fjamia.M2255 
47. Haux R, Ammenwerth E, Herzog W, Knaup P. Health care in the information society. A prognosis for the year 2013. International journal of medical informatics. 2002;66(1-3):3-21.Doi: 10.1016/s1386-5056(02)00030-8

48. Mikkelsen G, Aasly J. Concordance of information in parallel electronic and paper based patient records. Int J Med Inform. 2001;63(3):123-31.Doi: 10.1016/s1386-5056(01)00152-6

49. Shortliffe EH, Cimino J. Computer applications in health care and biomedicine. Springer, Heidelberg; 2006. https://link.springer.com/book/10.1007/978-1-4471-4474-8

50. Uslu AM, Stausberg J. Value of the electronic patient record: an analysis of the literature. Journal of biomedical informatics. 2008;41(4):675-82. Doi: 10.1016/j.jbi.2008.02.001

51. Young AS, Chaney E, Shoai R, Bonner L, Cohen AN, Doebbeling B, et al. Information technology to support improved care for chronic illness. J Gen Intern Med. 2007;22(3):425-30.Doi: $10.1007 /$ s11606-007-0303-4

52. Strollo BP, McClave SA, Miller KR. Complications of home enteral nutrition: mechanical complications and access issues in the home setting. Nutr Clin Pract. 2017;32(6):723-9. Doi: $10.1177 / 0884533617734529$

53. Metheny N. Initial and ongoing verification of feeding tube placement in adults (applies to blind insertions and placements with an electromagnetic device). Crit Care Nurse. 2016;36(2):E8-E13. Doi: $10.4037 / \mathrm{ccn} 2016141$

54. Klek S, Szybinski P, Sierzega M, Szczepanek K, Sumlet M, Kupiec M, et al. Commercial enteral formulas and nutrition support teams improve the outcome of home enteral tube feeding. JPEN J Parenter Enteral Nutr. 2011;35(3):380-5. Doi: 10.1177/0148607110378860

55. Franca SC, de Paiva SAR, Borgato MH, Fontes CMB, Simonetti JP, Lima SAM, et al. Homemade diet versus diet industrialized for patients using alternative feeding tube at home-An integrative review. Nutr Hosp. 2017;34(5):1281-7. Doi:10.20960/nh.1301

56. Moher D, Liberati A, Tetzlaff J, Altman DG, Group P. Preferred reporting items for systematic reviews and meta-analyses: the PRISMA statement. PLoS med. 2009;6(7):e1000097. Doi:10.1371/journal.pmed.1000097 